\title{
Algorithm of the arithmetic-geometric mean and its complex limits
}

(Dedicated to Professor Rentaro Agemi on his sixtieth birthday)

\author{
Kimimasa NisHIWADA
}

(Received May 27, 1996)

\begin{abstract}
The algorithm of the arithmetic-geometric mean defines a sequence converging to its complex limit. We prove that the correspondence between those sequences and their nonzero limits is one-to-one. Our method utilizes a new proof of a certain structural theorem of the arithmetic-geometric mean. In the process, our proof clarifies the relationship of the three aspects of our problem: patterns of the algorithm leading to various arithmetic-geometric means, a subgroup of $\pi_{1}(\mathbb{C} \backslash\{0,1\})$ and the modular group $\Gamma_{2}(4)$.
\end{abstract}

Key words: arithmetic-geometric mean, modular group.

\section{Introduction and the main result}

The study of the arithmetic-geometric mean of two complex numbers was started by Gauss. However, its various aspects were brought to light only by later mathematicians (e.g. von David [7] and Geppert [2]). Especially in Cox [1] was given a thorough exposition on a structural theorem of the complex means, as well as a good account of its historical background. In the present paper we are concerned with the same subject, but focusing our attention on a question not touched in the above works.

Let us begin with the famous algorithm leading to an arithmetic-geometric mean. With complex numbers $a$ and $b$ we consider

$$
a_{0}=a, \quad b_{0}=b,
$$

$$
a_{n}=\frac{a_{n-1}+b_{n-1}}{2}, \quad b_{n}=\left(a_{n-1} b_{n-1}\right)^{1 / 2}, \quad n=1,2, \ldots .
$$

A sequence $\left\{\left(a_{n}, b_{n}\right)\right\}(n=0,1, \ldots)$ is called an agm-sequence for $(a, b)$, if it satisfies the above algorithm. Because of two possible choices of $b_{n}$ at every step of the algorithm there are infinitely many such agm-sequences for $(a, b)$. It is well known that for any agm-sequence $\left\{\left(a_{n}, b_{n}\right)\right\}$ both sequences 
$\left\{a_{n}\right\}$ and $\left\{b_{n}\right\}$ converge to the same limit. In this paper this value will be also called the limit of the agm-sequence.

Now we ask the following question: Is the correspondence between agmsequences and their limits one-to-one or not? An answer to this question is given in the following theorem.

Theorem 1 Suppose that complex numbers $a$ and $b$ satisfy $a b \neq 0$ and $a \pm b \neq 0$. Let $\left\{\left(a_{n}, b_{n}\right)\right\}$ and $\left\{\left(a_{n}^{\prime}, b_{n}^{\prime}\right)\right\}$ be arbitrary agm-sequences for $(a, b)$ and $\tau$ and $\tau^{\prime}$ be their respective limits. If $\tau=\tau^{\prime} \neq 0$, then the two agm-sequences become identical, namely

$$
\left(a_{n}, b_{n}\right)=\left(a_{n}^{\prime}, b_{n}^{\prime}\right) \quad \text { for every } n \geq 0 .
$$

The above one-to-one correspondence would not be expected should we include agm-sequences converging to 0 . As pointed out in Cox [1], those agm-sequences that have non-zero limits form a countable set and therefore, one might say, "most" of agm-sequences converge to 0 . (We will review some basic properties of the agm-sequence in the next section.)

Our proof of the above theorem is strongly connected to the main result of Cox [1], which states that any non-zero arithmetic-geometric mean is expressed by two kinds of so called the simplest mean. His proof employs certain theta identities and in this sense agrees with the traditional treatment of the subject along the line of Gauss and Geppert (see Geppert [2]). However, the presence of theta identities seems (at least to the present author) to make the scheme of arguments somewhat unclear. Therefore, in proving our theorem, we prefer to follow a more elementary, analytical approach developed in [4].

This paper consists of the following two parts; in the first part (from Section 2 to Section 5) we will give a different proof to the main theorem of Cox [1] and in the second (Section 6) prove Theorem 1 using the information derived from the first part.

I would like to thank Prof. Masaaki Yoshida for informing me that the map $\mu_{3}$ in 6.2) is injective, one of the crucial facts in proving Theorem 1. My gratitude also goes to the referee, whose comments helped to improve various parts of this paper, especially the proof of Prop. 2.7. 


\section{Basic properties}

In this section we make a brief review of some basic properties of the complex arithmetic-geometric mean, mostly following von David [7] and Cox [1]. Throughout this paper, unless otherwise specified, the complex numbers $a$ and $b$ are supposed to satisfy the condition:

$$
a b \neq 0, \quad a \pm b \neq 0 .
$$

The following proposition states that, if the starting point $(a, b)$ satisfies (A), then, at every step of the algorithm $(\mathrm{AG}),\left(a_{n}, b_{n}\right)$ also satisfies the same condition.

Proposition 2.1 $\left(a_{n}, b_{n}\right)$ satisfies $(\mathrm{A})$ if and only if $\left(a_{n-1}, b_{n-1}\right)$ satisfies (A).

Proof. The proof is an easy consequence of the equality:

$$
a_{n} \pm b_{n}=\frac{1}{2}\left(\sqrt{a_{n-1}} \pm \sqrt{b_{n-1}}\right)^{2} \text {. }
$$

Definition 2.2 At each step of the algorithm $(\mathrm{AG}),\left(a_{n}, b_{n}\right)$ is called the right choice if the following holds:

$$
\operatorname{Re}\left(\frac{b_{n}}{a_{n}}\right)>0, \text { or } \operatorname{Re}\left(\frac{b_{n}}{a_{n}}\right)=0, \quad \operatorname{Im}\left(\frac{b_{n}}{a_{n}}\right)>0 .
$$

It is obvious that one of the two pairs $\left(a_{n}, \pm b_{n}\right)$ is the right choice, while the other is not.

The following estimates will be useful in proving the convergence of an $\operatorname{agm}$-sequence. Let $\operatorname{ang}(s, t)$ denote the unoriented angle between complex numbers $s$ and $t$, and put $M_{n}=\max \left(\left|a_{n}\right|,\left|b_{n}\right|\right)$.

Lemma 2.3 (i) If $\left(a_{n}, b_{n}\right)$ is the right choice, then

$$
\operatorname{ang}\left(a_{n}, b_{n}\right) \leq \frac{1}{2} \operatorname{ang}\left(a_{n-1}, b_{n-1}\right) .
$$

(ii) If $\left(a_{n}, b_{n}\right)$ is the right choice, then

$$
\left|a_{n}-b_{n}\right| \leq \frac{1}{2}\left|a_{n-1}-b_{n-1}\right| .
$$


(iii) For any choice, we have

$$
M_{n} \leq M_{n-1} .
$$

(iv) If $\left(a_{n}, b_{n}\right)(n \geq 1)$ is not the right choice, then

$$
M_{n+2} \leq \frac{3}{4} M_{n-1}
$$

For the proof of Lemma 2.3 we refer to the proof of Prop. 2.1 in Cox [1]. The above lemma enables us to prove the convergence of any agmsequence.

Proposition 2.4 Let $a$ and $b$ be arbitrary complex numbers and $\left\{\left(a_{n}, b_{n}\right)\right\}$ an agm-sequence for $(a, b)$. Then both sequences $\left\{a_{n}\right\}$ and $\left\{b_{n}\right\}$ converge to the same limit.

Proof. If $(a, b)$ does not satisfy the condition (A), then it is easy to show that the following are the only possibilities: either $a_{n}=b_{n}$ for every $n$, or that $b_{n}=0, n \geq n_{0}$, with a certain number $n_{0}$. In either case it is clear that both sequences converge to the same limit.

We now assume that $(a, b)$ satisfies (A). Note that $\left\{M_{n}\right\}$ is convergent due to (2.5). If $\left(a_{n}, b_{n}\right)$ is not the right choice for infinitely many numbers $n$, (2.6) implies $\lim M_{n}=0$. Hence $\lim a_{n}=\lim b_{n}=0$.

If there is a number $N$ such that $\left(a_{n}, b_{n}\right), \forall n \geq N$, is the right choice, then using (2.4) we get for any $k$

$$
\left|a_{N+k+1}-a_{N+k}\right| \leq \frac{1}{2}\left|a_{N+k}-b_{N+k}\right| \leq \frac{1}{2^{k+1}}\left|a_{N}-b_{N}\right|,
$$

which shows that $\left\{a_{n}\right\}$ is a Cauchy sequence. Using (2.4) again we have $\lim a_{n}=\lim b_{n}$.

Definition 2.5 Let $(a, b)$ satisfy $(\mathrm{A})$. We denote by $\mathfrak{M}(a, b)$ the set of all non-zero limits of agm-sequences $\left\{\left(a_{n}, b_{n}\right)\right\}$ for $(a, b)$. For the agm-sequence having the property that $\left(a_{n}, b_{n}\right)$ is the right choice for every $n \geq 1$, its limit is called the simplest mean of $a$ and $b$ and denoted by $M(a, b)$.

The following proposition shows that $M(a, b) \in \mathfrak{M}(a, b)$ and also that $\mathfrak{M}(a, b)$ is a countable set. Its proof can be found in Cox [1, pages 286-287].

Proposition 2.6 The limit of an agm-sequence is not equal to 0 if and 
only if $\left(a_{n}, b_{n}\right)$ is the right choice for all but finitely many $n$.

In view of (2.6) one can surmise that, the more non-right choices appear in an agm-sequence, the smaller its limit becomes. However, it also matters where a non-right choice appears.

Proposition 2.7 For any $\delta \geq 0$, there is a number $n_{0}$ such that, for any agm-sequence $\left\{\left(a_{n}, b_{n}\right)\right\}$ for $(a, b)$ satisfying

$$
\lim M_{n} \geq \delta
$$

$\left(a_{n}, b_{n}\right)$ is the right choice for every $n \geq n_{0}$.

Proof. Take any agm-sequence $\left\{\left(a_{n}, b_{n}\right)\right\}$ satisfying (2.8). From (2.6) it is clear that the number of non-right choices appearing in the sequence does not exceed a certain number depending on $\delta$, say, $U(\delta)$.

In order to prove our proposition, it suffices to show the following; if $\left(a_{m+j}, b_{m+j}\right), 0 \leq j \leq N-1$, are right choices, while $\left(a_{m+N}, b_{m+N}\right)$ is not, then $N$ is bounded by a number depending on $\delta$. Suppose that this was false, $N$ being taken arbitrarily large.

Put $M=\max (|a|,|b|)$. (2.4) implies

$$
\begin{aligned}
& \left|a_{m+N+1}\right|=\left|\frac{a_{m+N}-\left(-b_{m+N}\right)}{2}\right| \leq \frac{1}{2^{N+1}}\left|a_{m}-b_{m}\right| \leq \frac{M}{2^{N}}, \\
& \left|b_{m+N+1}\right| \leq M .
\end{aligned}
$$

We get further

$$
\begin{aligned}
\left|b_{m+N+2}\right| & \leq\left(M \cdot \frac{M}{2^{N}}\right)^{1 / 2}=\frac{M}{2^{N / 2}} \\
\left|b_{m+N+3}\right| & \leq\left(M \cdot \frac{M}{2^{N / 2}}\right)^{1 / 2}=\frac{M}{2^{N / 2^{2}}}, \\
& \vdots \\
\left|b_{m+N+k+1}\right| & \leq \frac{M}{2^{N / 2^{k}}}
\end{aligned}
$$

for any $k$.

Now choose a number $l_{0}$ such that $\frac{M}{2^{l_{0}-1}}<\frac{\delta}{2}$ and pick $k>l_{0}(U(\delta)+1)$. It follows that any sequence of $k$ elements contain a sequence consisting of 
$l_{0}$ right choices. At the last term $\left(a_{p}, b_{p}\right)$ of such sequence we have

$$
\begin{aligned}
\left|b_{p}\right| & \leq \frac{M}{2^{N / 2^{k}}}, \\
\left|a_{p}-b_{p}\right| & \leq \frac{1}{2^{l_{0}}}\left|a_{p-l_{0}}-b_{p-l_{0}}\right| \leq \frac{M}{2^{l_{0}-1}}
\end{aligned}
$$

and therefore

$$
\left|a_{p}\right| \leq \frac{M}{2^{l_{0}-1}}+\frac{M}{2^{N / 2^{k}}} .
$$

Now take $N$ so large that $\frac{M}{2^{N / 2^{k}}}<\delta / 2$. Then $(2.9)$ and $(2.10)$ imply $M_{p}<\delta$, a contradiction to (2.8).

The above proposition shows that there are only finitely many agmsequences satisfying (2.8). Therefore we get

Corollary 2.8 [von David [7]] The point 0 is the only limit point of the set $\mathfrak{M}(a, b)$.

As a much finer structural theorem of $\mathfrak{M}(a, b)$ we know

Theorem 2.9 [Cox [1]] Suppose that $a$ and $b$ satisfy (A) and $|a| \geq|b|$. Then a complex number $\tau$ belongs to $\mathfrak{M}(a, b)$ if and only if the following expression holds.

$$
\frac{1}{\tau}=\frac{p}{M(a, b)}+i \frac{q}{M(a+b, a-b)},
$$

where $p$ and $q$ are arbitrary relatively prime integers satisfying $p \equiv 1 \bmod$ 4 and $q \equiv 0 \bmod 4$.

Part of the above theorem was already known by Gauss (as its circumstantial evidence given in Cox [1]), but a precise statement and a proof of the theorem were given in much later time. Cox' proof employs the theta functions

$$
p(\tau)=\sum e^{i \pi \tau n^{2}}, \quad q(\tau)=\sum(-1)^{n} e^{i \pi \tau n^{2}}, \quad \operatorname{Im} \tau \geq 0 .
$$

and the fact that, if $a / b=p(\tau)^{2} / q(\tau)^{2}$, then

$$
\mu=\frac{a}{p(\tau)^{2}} \in \mathfrak{M}(a, b)
$$


We will prove the theorem without relying on theta functions. In our proof the following integral expressions of $M(a, b)$ and $M(a+b, a-b)$ will play an important role. The first formula is well known, as it is cited in Cox [1] in a slightly different form. The second seems to have evaded due attention.

Theorem 2.10 Suppose $0<b<a$. Then the following integral formulas are valid on the elliptic curve $y^{2}=x(1-x)\left(a^{2}(1-x)+b^{2} x\right)$.

$$
\begin{aligned}
\frac{1}{M(a, b)} & =\frac{1}{\pi} \int_{0}^{1} \frac{d x}{y} \quad(y>0), \\
\frac{i}{M(a+b, a-b)} & =\frac{1}{\pi} \int_{0}^{-\infty} \frac{d x}{y} \quad(\operatorname{Im} y>0) .
\end{aligned}
$$

Proof. Let $\left\{\left(a_{n}, b_{n}\right)\right\}$ be the agm-sequence for $(a, b)$ consisting only of right choices. Define the new variable $x^{\prime}$ by

$$
\sqrt{x}=\frac{2 a \sqrt{x^{\prime}}}{a+b+(a-b) x^{\prime}} .
$$

A straightforward computation shows

$$
\frac{d x}{\left\{x(1-x)\left(a^{2}(1-x)+b^{2} x\right)\right\}^{1 / 2}}=\frac{d x^{\prime}}{\left\{x^{\prime}\left(1-x^{\prime}\right)\left(a_{1}^{2}\left(1-x^{\prime}\right)+b_{1}^{2} x^{\prime}\right)\right\}^{1 / 2}} .
$$

A successive use of similar variable changes ensures that the right-hand side of (2.12) equals

$$
\begin{aligned}
\lim _{n \rightarrow \infty} \frac{1}{\pi} \int_{0}^{1} & \frac{d x}{\left\{x(1-x)\left(a_{n}^{2}(1-x)+b_{n}^{2} x\right)\right\}^{1 / 2}} \\
& =\frac{1}{\pi M(a, b)} \int_{0}^{1} \frac{d x}{\{x(1-x)\}^{1 / 2}}=\frac{1}{M(a, b)}
\end{aligned}
$$

which completes the proof of $(2.12)$.

Now by another change of the variable, $(1-x)\left(1-x^{\prime}\right)=1$, we have

$$
\begin{aligned}
\int_{0}^{-\infty} \frac{d x}{\left\{x(1-x)\left(a^{2}(1-x)+b^{2} x\right)\right\}^{1 / 2}} \\
\quad=i \int_{0}^{1} \frac{d x^{\prime}}{\left\{x^{\prime}\left(1-x^{\prime}\right)\left(a^{2}\left(1-x^{\prime}\right)+\left(a^{2}-b^{2}\right) x^{\prime}\right)\right\}^{1 / 2}} .
\end{aligned}
$$


In view of (2.12) the above integral is equal to

$$
\frac{i}{M\left(a, \sqrt{a^{2}-b^{2}}\right)}=\frac{i}{M(a+b, a-b)},
$$

which completes the proof of the theorem.

\section{Analyticity of arithmetic-geometric means}

The obvious homogeneity

$$
M(a, b)=a M\left(1, \frac{b}{a}\right), \quad \mathfrak{M}(a, b)=a \mathfrak{M}\left(1, \frac{b}{a}\right)
$$

may justify our temporary restriction $a=1, b=z \in \mathbb{C}$. We will write $a_{n}=a_{n}(z)$ and $b_{n}=b_{n}(z)$. The condition (A) now becomes

$$
z \in \mathbb{C}_{0}:=\mathbb{C} \backslash\{0, \pm 1\} .
$$

In this section we will show that every value of $\mathfrak{M}(1, z)$ can be considered a germ of a holomorphic function of $z$ and that such a holomorphic function can be reached by analytic continuation from any single value of $\mathfrak{M}(1, z)$, for example from $M(1, z)$.

Let us first note that $a_{n}(z)$ and $b_{n}(z)$ can be analytically continued to (multi-valued) algebraic functions of $z$ by the relationship (AG). Proposition 2.1 indicates that possible singularities of these algebraic functions are 0 and \pm 1 . This can be paraphrased in the following way.

Proposition 3.1 Let $z$ and $z^{\prime}$ be points of $\mathbb{C}_{0}$ and $\gamma$ be a continuous curve in $\mathbb{C}_{0}$ joining $z$ with $z^{\prime}$. Denote by $(\gamma)_{*}$ the analytic continuation along the curve $\gamma$. If $\left\{\left(a_{n}(z), b_{n}(z)\right)\right\}$ is an agm-sequence for $(1, z)$, then $\left\{\left((\gamma)_{*} a_{n}\left(z^{\prime}\right),(\gamma)_{*} b_{n}\left(z^{\prime}\right)\right)\right\}$ becomes an agm-sequence for $\left(1, z^{\prime}\right)$.

Note that it does not necessarily follow that $\left((\gamma)_{*} a_{n}\left(z^{\prime}\right),(\gamma)_{*} b_{n}\left(z^{\prime}\right)\right)$ is the right choice even if $\left(a_{n}(z), b_{n}(z)\right)$ is so. Under certain circumstances, however, one may expect something like this to happen.

Proposition 3.2 Let $U$ be a connected set in $\mathbb{C}_{0}$ and $\left\{\left(a_{n}(z), b_{n}(z)\right)\right\}$ be an agm-sequence defined in $U$ in the sense that each $a_{n}$ and $b_{n}(n=0,1, \cdots)$ are single-valued continuous functions of $z$ in $U$. Suppose that there exists a number $N$ and a point $z_{0} \in U$ such that

$$
\left(a_{N}(z), b_{N}(z)\right), \quad \forall z \in U, \text { the right choice, }
$$


$\left(a_{n}\left(z_{0}\right), b_{n}\left(z_{0}\right)\right), \quad \forall n \geq N$, the right choice.

It follows then that

$$
\left(a_{n}(z), b_{n}(z)\right), \quad \forall z \in U, \quad \forall n \geq N, \quad \text { the right choice. }
$$

Proof. Suppose that the conclusion of the proposition were false. Then there would be a number $m>N$ such that $\left(a_{m}(z), b_{m}(z)\right)$ is not the right choice for some $z \in U$. Let $m_{0}$ be the minimum of such $m$.

Consider a curve in $U$ connecting $z_{0}$ with a point $z$ where $\left(a_{m_{0}}(z), b_{m_{0}}(z)\right)$ is not the right choice. On this curve there must be a point $z_{1}$ satisfying $\operatorname{Re}\left[b_{m_{0}}\left(z_{1}\right) / a_{m_{0}}\left(z_{1}\right)\right]=0$, which means $\operatorname{ang}\left(a_{m_{0}}\left(z_{1}\right), b_{m_{0}}\left(z_{1}\right)\right)=\pi / 2$. Then the next lemma shows that $\left(a_{m_{0}-1}\left(z_{1}\right), b_{m_{0}-1}\left(z_{1}\right)\right)$ is not the right choice, a contradiction to our assumption that $m_{0}$ be minimal.

Lemma 3.3 If $\operatorname{ang}\left(a_{n}, b_{n}\right)=\pi / 2$, then $\operatorname{ang}\left(a_{n-1}, b_{n-1}\right)=\pi$ and in particular $\left(a_{n-1}, b_{n-1}\right)$ is not the right choice.

Proof. We should only note that $a_{n-1}$ and $b_{n-1}$ are the solutions of the equation $X^{2}-2 a_{n} X+b_{n}^{2}=0$.

The results so far obtained now enable us to prove the analyticity of arithmetic-geometric means in $\mathbb{C}_{0}$.

Theorem 3.4 Let $\left\{\left(a_{n}\left(z_{0}\right), b_{n}\left(z_{0}\right)\right)\right\}$ be an agm-sequence at $z_{0} \in \mathbb{C}_{0}$ with its limit $\tau\left(z_{0}\right) \in \mathfrak{M}\left(1, z_{0}\right)$. Then there exists a neighborhood $U$ of $z_{0}$ such that, if $\left(a_{n}(z), b_{n}(z)\right)$ is the analytic extension into $U$ of $\left(a_{n}\left(z_{0}\right), b_{n}\left(z_{0}\right)\right)$ by the relation $(\mathrm{AG})$, then

$$
\tau(z):=\lim _{n \rightarrow \infty} a_{n}(z)=\lim _{n \rightarrow \infty} b_{n}(z)
$$

is non-zero and holomorphic in $U$.

Proof. Since $\tau\left(z_{0}\right) \in \mathfrak{M}\left(1, z_{0}\right)$, there exists a number $N$ such that $\left(a_{n}\left(z_{0}\right), b_{n}\left(z_{0}\right)\right)$ is the right choice for every $n \geq N$. We may also assume that $\operatorname{Re}\left[b_{N}\left(z_{0}\right) / a_{N}\left(z_{0}\right)\right]>0$. Take a neighborhood $U$ of $z_{0}$ so that $\left(a_{N}(z), b_{N}(z)\right)$ is the right choice for $z \in U$. Then Proposition 3.2 implies that $\left(a_{n}(z), b_{n}(z)\right)$ are right choices for all $z \in U$ and $n \geq N$. Therefore we know that $\tau(z) \neq 0$ belonging to $\mathfrak{M}(1, z)$. To prove that $\tau(z)=\lim a_{n}(z)$ is holomorphic, we have only to notice its uniform convergence derived from 
$(2.7)$ :

$$
\begin{aligned}
\left|a_{N+k+1}(z)-a_{N+k}(z)\right| & \leq \frac{1}{2}\left|a_{N+k}(z)-b_{N+k}(z)\right| \\
& \leq \frac{1}{2^{k+1}}\left|a_{N}(z)-b_{N}(z)\right| .
\end{aligned}
$$

The following two propositions pave the way for our goal in this section that all values of $\mathfrak{M}(1, z)$ are connected by analytic continuation.

Proposition 3.5 Let $\left\{\left(a_{k}\left(z_{0}\right), b_{k}\left(z_{0}\right)\right)\right\}(k=0,1, \ldots)$ be an agm-sequence at $z_{0} \in \mathbb{C}_{0}$. Let $\gamma:[0,1] \rightarrow \mathbb{C}_{0}$ be a continuous curve in $\mathbb{C}_{0}$ satisfying

$$
\gamma(0)=\frac{b_{n}}{a_{n}}\left(z_{0}\right)
$$

for some number $n$. Then there exists a curve $\tilde{\gamma}:[0,1] \rightarrow \mathbb{C}_{0}$ such that

$$
\frac{b_{n}}{a_{n}}(\tilde{\gamma}(s))=\gamma(s), \quad 0 \leq s \leq 1, \quad \tilde{\gamma}(0)=z_{0},
$$

where the left hand side of the equality indicates the analytic continuation of the function $b_{n} / a_{n}$ along the curve $\tilde{\gamma}$.

Proof. If $n=0$, the proposition is trivial as we can put $\tilde{\gamma}=\gamma$. As the induction hypothesis, suppose that it is true up to $n-1$.

Put

$$
c_{0}=\frac{a_{n}}{b_{n}}\left(z_{0}\right), \quad c_{1}=\frac{a_{n-1}}{b_{n-1}}\left(z_{0}\right)
$$

The two values $w_{n}=b_{n} / a_{n}$ and $w_{n-1}=b_{n-1} / a_{n-1}$ are related by

$$
w_{n}=\frac{\sqrt{a_{n-1} b_{n-1}}}{\left(a_{n-1}+b_{n-1}\right) / 2}=\frac{2 \sqrt{w_{n-1}}}{1+w_{n-1}},
$$

or equivalently

$$
w_{n}^{2} w_{n-1}^{2}+2\left(w_{n}^{2}-2\right) w_{n-1}+w_{n}^{2}=0 .
$$

As a quadratic equation of $w_{n-1}$ it has its discriminant $D \neq 0$ for $w_{n} \in \mathbb{C}_{0}$. Each branch $w_{n} \rightarrow w_{n-1}$ defines a local homeomorphism. It is then easy to find a curve $\gamma_{1}$, which is a "lift" of $\gamma$ in the sense that the values

$$
w_{n}=\gamma(s), \quad w_{n-1}=\gamma_{1}(s) \quad(0 \leq s \leq 1)
$$


are related by (3.2) with their respective initial values $c_{0}$ and $c_{1}$.

Since our induction hypothesis ensures the existence of the curve $\tilde{\gamma}$ such that

$$
\frac{b_{n-1}}{a_{n-1}}(\tilde{\gamma}(s))=\gamma_{1}(s), \quad 0 \leq s \leq 1, \quad \tilde{\gamma}(0)=z_{0},
$$

the same curve satisfies

$$
\frac{b_{n}}{a_{n}}(\tilde{\gamma}(s))=\gamma(s), \quad 0 \leq s \leq 1,
$$

which completes our proof.

Proposition 3.6 Let $\left\{\left(a_{n}\left(z_{0}\right), b_{n}\left(z_{0}\right)\right)\right\}$ be an agm-sequence at $z_{0} \in \mathbb{C}_{0}$. Suppose that there is a number $N$ such that $\left(a_{n}\left(z_{0}\right), b_{n}\left(z_{0}\right)\right)$ is the right choice for every $n \geq N$. Then there exists a continuous curve $\gamma:[0,1] \rightarrow \mathbb{C}_{0}$ with $\gamma(0)=z_{0}$ and $\gamma(1)=z_{1}$, such that

$$
\left((\gamma)_{*} a_{n}\left(z_{1}\right),(\gamma)_{*} b_{n}\left(z_{1}\right)\right)
$$

becomes the right choice for every $n \geq N-1$. Here $(\gamma)_{*}$ denotes the analytic continuation along the curve $\gamma$.

Proof. If $\left(a_{N-1}\left(z_{0}\right), b_{N-1}\left(z_{0}\right)\right)$ is the right choice we may only put $\gamma(s)=$ $z_{0}(0 \leq s \leq 1)$.

Suppose now that it is not the right choice. In this case we have

$$
\operatorname{Re}\left(\frac{b_{N-1}}{a_{N-1}}\left(z_{0}\right)\right) \leq 0 .
$$

Let us choose a branch $\tau_{0}=\sqrt{b_{N-1}\left(z_{0}\right) / a_{N-1}\left(z_{0}\right)}$ satisfying

$$
\frac{b_{N}}{a_{N}}\left(z_{0}\right)=\frac{2 \sqrt{a_{N-1} b_{N-1}}}{a_{N-1}+b_{N-1}}=\frac{2 \tau_{0}}{1+\tau_{0}^{2}} .
$$

Since $\left(a_{N}\left(z_{0}\right), b_{N}\left(z_{0}\right)\right)$ is the right choice, we have

$$
\begin{aligned}
& \operatorname{Re}\left(\frac{1}{\tau_{0}+\tau_{0}^{-1}}\right)>0, \text { or } \operatorname{Re}\left(\frac{1}{\tau_{0}+\tau_{0}^{-1}}\right)=0, \\
& \operatorname{Im}\left(\frac{1}{\tau_{0}+\tau_{0}^{-1}}\right)>0
\end{aligned}
$$


or equivalently

$$
\operatorname{Re} \tau_{0}>0, \quad \text { or } \operatorname{Re} \tau_{0}=0, \quad\left(1-\left|\tau_{0}\right|^{2}\right) \operatorname{Im} \tau_{0}>0 .
$$

Now take a simple arc $\rho$ satisfying

$$
\begin{gathered}
\rho(0)=\frac{b_{N-1}}{a_{N-1}}\left(z_{0}\right), \quad|\rho(s)|=|\rho(0)| \quad(0 \leq s \leq 1) \\
\text { and } \quad \operatorname{Re} \rho(1)>0
\end{gathered}
$$

and lying in $\{z ; \operatorname{Im} z \geq 0\}$ if $\operatorname{Im} \tau_{0}>0$ and in $\{z ; \operatorname{Im} z \leq 0\}$ if $\operatorname{Im} \tau_{0}<0$ (see Fig. 3.1). Note that $\tau_{0}$ is not real by (3.3).

In view of Proposition 3.5 there is a curve $\gamma$ in $\mathbb{C}_{0}$ satisfying

$$
\frac{b_{N-1}}{a_{N-1}}(\gamma(s))=\rho(s), \quad 0 \leq s \leq 1, \quad \gamma(0)=z_{0} .
$$

We denote its end point by $\gamma(1)=z_{1}$ and along the curve consider the branch

$$
\tau(z)=\sqrt{\frac{b_{N-1}}{a_{N-1}}(z)}
$$

with its initial value $\tau\left(z_{0}\right)=\tau_{0}$. Since $\operatorname{Re} \tau(z)>0$ for $z=\gamma(s), s>0$, it
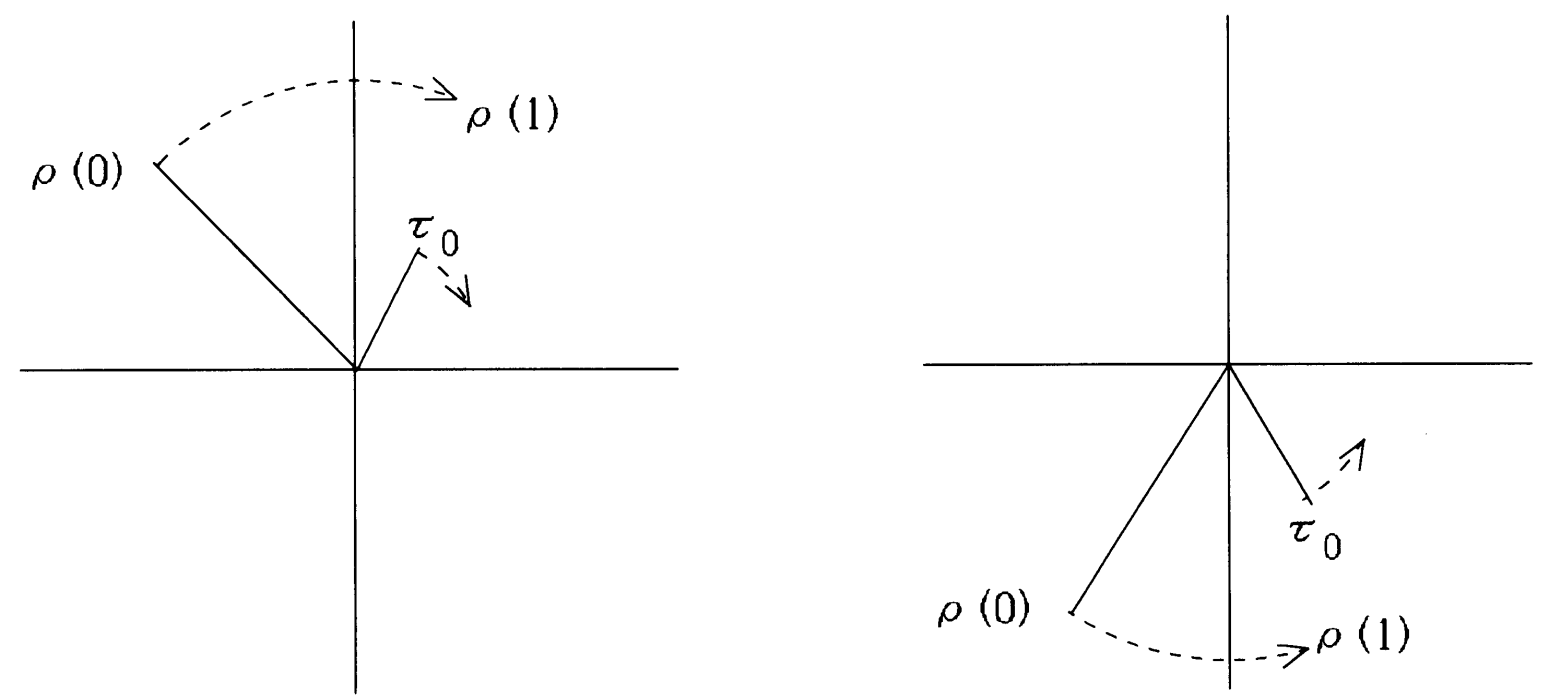

Fig. 3.1. 
follows that

$$
\operatorname{Re}\left(\frac{b_{N}}{a_{N}}(z)\right)=\operatorname{Re}\left(\frac{2}{\tau(z)+\tau(z)^{-1}}\right)>0, \quad z=\gamma(s), s>0 .
$$

In other words $\left(a_{N}(z), b_{N}(z)\right)$ is the right choice for any $z \in \gamma$.

This fact, combined with our hypothesis that $\left(a_{n}\left(z_{0}\right), b_{n}\left(z_{0}\right)\right)$ is the right choice for every $n \geq N$, allows us to apply Proposition 3.2 and conclude that $\left(a_{n}\left(z_{1}\right), b_{n}\left(z_{1}\right)\right)$ is the right choice for every $n \geq N$. Since $\left(a_{N-1}\left(z_{1}\right), b_{N-1}\left(z_{1}\right)\right)$ is also the right choice thanks to $(3.4)$, our proof is now complete.

$M(1, z)$, as an element of $\mathfrak{M}(1, z)$, defines a holomorphic germ at every point $z$ and can be analytically continued along any curve in $\mathbb{C}_{0}$. However its analytically continued value does not necessarily remain to be $M(1, z)$. We shall now briefly discuss the discontinuity of the function.

Proposition 3.7 Let $\left\{\left(c_{n}(z), d_{n}(z)\right)\right\}$ be the agm-sequence for $(1, z)$ consisting only of right choices with its limit $M(1, z)$. Then the point 1 is a removable singularity of the functions $M(1, z), c_{n}(z)$ and $d_{n}(z)$ for every $n$.

Proof. It is easy to prove by induction that $\lim _{z \rightarrow 1} c_{n}(z)=1$ and $\lim _{z \rightarrow 1} d_{n}(z)=1$ and that the functions $c_{n}$ and $d_{n}$ are holomorphic at 1. Since (2.5) indicates that $c_{n}$ and $d_{n}$ are uniformly bounded in a neighborhood of 1 , their limit $M(1, z)$ becomes holomorphic, too.

Let $\sqrt{z}$ be a branch such that $\arg \sqrt{z}=0$ when $\arg z=0$. In the closed region $-\pi \leq \arg z \leq \pi,\left(\frac{1+z}{2}, \sqrt{z}\right)$ is the right choice if and only if

$$
\begin{aligned}
z \in \mathbb{C}_{0}^{\prime}:=\{\arg z=-\pi,|z|>1\} & \cup\{-\pi<\arg z<\pi\} \\
\cup & \{\arg z=\pi,|z|<1\} .
\end{aligned}
$$

In view of Propositions 3.2 and 3.7 it follows that $M(1, z)$ is continuous in $\mathbb{C}_{0}^{\prime}$ and holomorphic in the interior of $\mathbb{C}_{0}^{\prime}$. In other words $M(1, z)$ is continuous from above on $\{z \in \mathbb{R} ;-1<z<0\}$ and from below on $\{z \in \mathbb{R} ; z<-1\}$.

For a later reference we should note that the above fact also describes a similar behavior of the function $M(1+z, 1-z)$; it is continuous from above on $\{z \in \mathbb{R} ; z<-1\}$ and from below on $\{z \in \mathbb{R} ; z>1\}$, being holomorphic in the rest of $\mathbb{C}_{0}$.

Let us now turn back to Proposition 3.6. Starting with any $\tau\left(z_{0}\right) \in$ $\mathfrak{M}\left(1, z_{0}\right), z_{0} \in \mathbb{C}_{0}$, and applying the proposition for sufficiently many times 
we can find a continuous curve $\gamma(s), 0 \leq s \leq 1$, with $\gamma(0)=z_{0}$ and $\gamma(1)=z_{1}$ such that

$$
(\gamma)_{*} \tau(z)=M(1, z) \text { for } z \text { near } z_{1}
$$

Connecting $z_{1}$ with $z_{0}$ by a path not crossing the real segment $(-\infty, 0)$, we obtain

Proposition 3.8 Let $z_{0} \in \mathbb{C}_{0}$. Every value of $\mathfrak{M}\left(1, z_{0}\right)$ is attained by the analytic continuation of $M(1, z)$ along a cycle in $\mathbb{C}_{0}$ through $z_{0}$.

\section{Analytic continuation of $M(1, z)$}

In this section we are concerned with the values obtained by analytic continuation of $M(1, z)$ along various cycles through $z \in \mathbb{C}_{0}$. For a while we assume $0<z<1$. This condition will be removed at the end of this section.

The integral expressions (2.12) and (2.13) now read as

$$
\begin{aligned}
\frac{1}{M(1, z)} & =\frac{1}{\pi} \int_{0}^{1} \frac{d x}{y} \\
\frac{i}{M(1+z, 1-z)} & =\frac{1}{\pi} \int_{0}^{-\infty} \frac{d x}{y} .
\end{aligned}
$$

with $y^{2}=x(1-x)\left(1-\left(1-z^{2}\right) x\right)$.

Let us define three circles $C_{-1}, C_{0}$ and $C_{1}$, their centers being located respectively at points $-1,0$ and 1 , their radii being respectively $1-z, z$ and $1-z$. Note that each circle touches one or both of the points $z$ and $-z$.

Using these circles we now define cycles $\gamma_{-1}, \gamma_{0}$ and $\gamma_{1}$, all through $z$, in the following manner. $\gamma_{1}$ starts from $z$ moving counterclockwise once along $C_{1} \cdot \gamma_{0}$ starts from $z$ tracing the circle $C_{0}$ once counterclockwise. $\gamma_{-1}$ is the curve starting from $z$, moving to $-z$ along the upper half of $C_{0}$, then encircling once the point -1 counterclockwise along $C_{-1}$ and coming back from $-z$ to $z$ on the same upper part of $C_{0}$.

The three elements of the fundamental group $\pi_{1}\left(\mathbb{C}_{0}, z\right)$ corresponding to $\gamma_{-1}, \gamma_{0}$ and $\gamma_{1}$ will be denoted by the same symbols. It is well known that it is a free group generated by these elements.

Now consider the map

$$
\varphi: \mathbb{C}_{0} \longrightarrow \mathbb{C}_{1}:=\mathbb{C} \backslash\{0,1\}
$$


defined by $\varphi(\zeta)=1 /\left(1-\zeta^{2}\right)$. Putting $\lambda=1 /\left(1-z^{2}\right)$, we obtain the induced homomorphism

$$
\varphi_{*}: \pi_{1}\left(\mathbb{C}_{0}, z\right) \longrightarrow \pi_{1}\left(\mathbb{C}_{1}, \lambda\right) .
$$

The definition of $\gamma_{j}(j=0, \pm 1)$ implies that the images of $\gamma_{j}$ by $\varphi_{*}$ can be written as:

$$
\varphi_{*} \gamma_{1}=\delta_{\infty}^{-1}, \quad \varphi_{*} \gamma_{0}=\delta_{1}^{2}, \quad \varphi_{*} \gamma_{-1}=\delta_{1}^{-1} \delta_{\infty}^{-1} \delta_{1} .
$$

Here $\delta_{1}$ corresponds to a Jordan curve in $\mathbb{C}_{1}$ through $\lambda$ such that it encircles the point 1 but leaves the point 0 outside, while $\delta_{\infty}$ corresponds to a Jordan curve encircling both 0 and 1 . Both curves are oriented in the positive sense.

We will rewrite (4.1) and (4.2) with $\lambda$ now introduced:

$$
\pi M(1, z)^{-1}=u_{1}(\lambda), \quad i \pi M(1+z, 1-z)^{-1}=u_{2}(\lambda),
$$

where $u_{1}$ and $u_{2}$ are defined by the following integrals

$$
\begin{aligned}
& u_{1}(\lambda)=\sqrt{\lambda} \int_{0}^{1} \frac{d t}{s} \\
& u_{2}(\lambda)=\sqrt{\lambda} \int_{0}^{-\infty} \frac{d t}{s}
\end{aligned}
$$

on the elliptic curve

$$
E: s^{2}=t(1-t)(\lambda-t)
$$

Our objective is to evaluate the change of integral values (4.1) and (4.2) after having $z$ move along the cycles $\gamma_{j}, j=0, \pm 1$. This question may be equivalently posed as the evaluation of $u_{1}$ and $u_{2}$ when $\lambda$ moves along $\delta_{1}$ and $\delta_{\infty}$.

Now the elliptic curve $E$ is supposed to consist of two copies $E_{1}$ and $E_{2}$ of the Riemann sphere $\mathbb{C} \cup\{\infty\}$, in such a way that $E=E_{1} \cup E_{2}$ with both $E_{1}$ and $E_{2}$ having cuts along the real intervals $(0,1)$ and $(\lambda, \infty)$, where the upper and lower edges of the cuts of $E_{1}$ are identified respectively with the lower and upper edges of the corresponding cuts of $E_{2}$. The integration path in (4.6) is supposed to lie on the upper edge of $(0,1)$ in $E_{1}$, while the path in (4.7) to lie in $E_{1}$.

When $\lambda$ moves along $\delta_{1}$, the integration path for $u_{1}$, after being deformed as indicated in Fig. 4.1, will be changed to a path as described in 


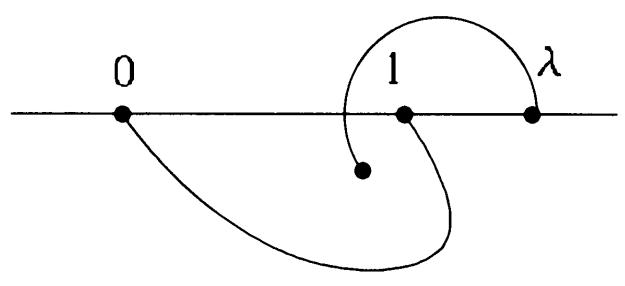

Fig. 4.1.

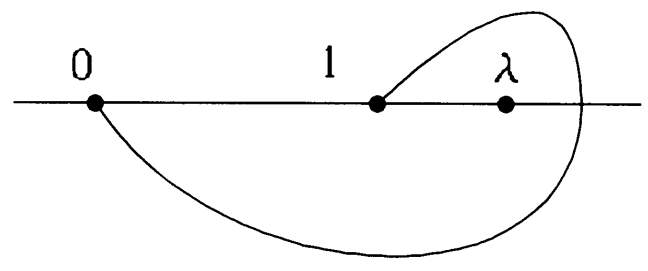

Fig. 4.2 .

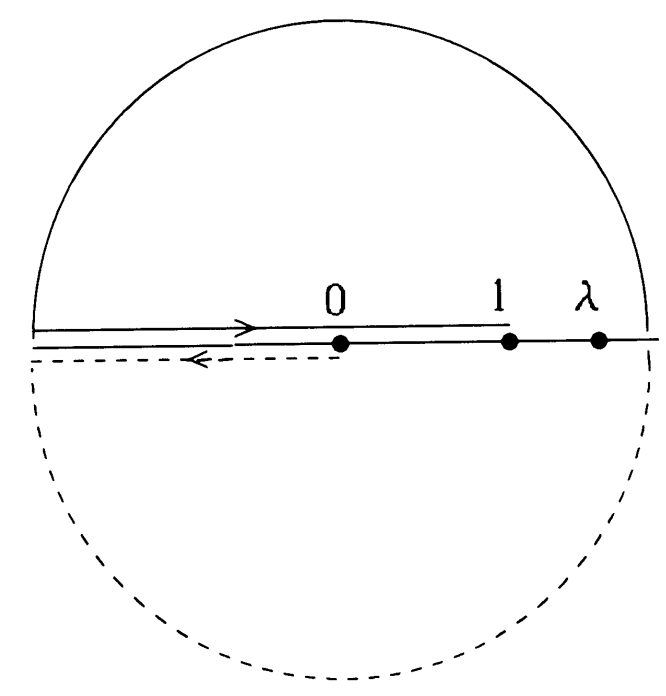

Fig. 4.3.

Fig. 4.2. The new path has its homotopic equivalence suggested in Fig. 4.3, where the real curve indicates a path in $E_{1}$ and the dotted one in $E_{2}$.

Since $d t / s$ is a holomorphic differential form in $E$ and in particular at the point $\infty$, the integration around $\infty$ can be made as small as we would like. Counting also that $\sqrt{\lambda}$ is un-changed by the curve $\delta_{1}$, we arrive at the formula

$$
\left(\delta_{1}\right)_{*} u_{1}=u_{1}-2 u_{2}, \quad\left(\delta_{1}\right)_{*} u_{2}=u_{2} .
$$

A similar observation leads to

$$
\left(\delta_{\infty}\right)_{*} u_{1}=u_{1}, \quad\left(\delta_{\infty}\right)_{*} u_{2}=-2 u_{1}+u_{2} .
$$

Therefore the circuit matrices of $\left(u_{1}, u_{2}\right)$ along $\delta_{1}$ and $\delta_{\infty}$ are respec- 
tively $U^{-1}$ and $V^{-1}$, where

$$
U=\left(\begin{array}{ll}
1 & 2 \\
0 & 1
\end{array}\right), \quad V=\left(\begin{array}{ll}
1 & 0 \\
2 & 1
\end{array}\right)
$$

Denoting the circuit matrix of $\left(M(1, z)^{-1}, i M(1+z, 1-z)^{-1}\right)$ along $\gamma \in \pi_{1}\left(\mathbb{C}_{0}, z\right)$ by $\mu(\gamma)$, we have in view of $(4.4)$

$$
\begin{gathered}
\mu\left(\gamma_{1}\right)=V=\left(\begin{array}{ll}
1 & 0 \\
2 & 1
\end{array}\right) \\
\mu\left(\gamma_{0}\right)=U^{-2}=\left(\begin{array}{cc}
1 & -4 \\
0 & 1
\end{array}\right) \\
\mu\left(\gamma_{-1}\right)=U V U^{-1}=\left(\begin{array}{cc}
5 & -8 \\
2 & -3
\end{array}\right)
\end{gathered}
$$

Let $\Gamma$ be the subgroup of $S L(2, \mathbb{Z})$ generated by $V, U^{-2}$ and $U V U^{-1}$. The homomorphism induced from $(4.11) \sim(4.13)$ will be also denoted by $\mu$ :

$$
\mu: \pi_{1}\left(\mathbb{C}_{0}, z\right) \longrightarrow \Gamma \subset S L(2, \mathbb{Z})
$$

indicating the circuit matrix $\mu(\gamma)$ for any $\gamma \in \pi_{1}\left(\mathbb{C}_{0}, z\right)$.

The map can be considered a monodromy representation of a certain second order equation having $\left(M(1, z)^{-1}, i M(1+z, 1-z)^{-1}\right)$ as its fundamental solution, though this fact will not be used in this paper.

If we write

$$
\mu(\gamma)=\left(\begin{array}{ll}
p & q \\
r & s
\end{array}\right), \quad \gamma \in \pi_{1}\left(\mathbb{C}_{0}, z\right),
$$

then it follows that

$$
(\gamma)_{*} M(1, z)^{-1}=p M(1, z)^{-1}+i q M(1+z, 1-z)^{-1}
$$

Until now we have assumed $0<z<1$. This restriction, however, can be easily removed. Indeed suppose that $z \in \mathbb{C}_{0} \backslash\{t \in \mathbb{R} ; t<-1\}$. Following the discussion made below (3.5) we can find a continuous curve $\rho$ joining $z$ with $z_{0} \in\{t \in \mathbb{R} ; 0<t<1\}$ such that both $M(1, z)$ and $M(1+z, 1-z)$ are continuous on $\rho$. Then for any cycle $\gamma$ in $\mathbb{C}_{0}$ through $z, \gamma^{\prime}=\rho \gamma \rho^{-1}$ is a 
cycle through $z_{0}$. Therefore

$$
\begin{aligned}
(\gamma)_{*} M(1, z)^{-1} & =\left(\rho^{-1}\right)_{*}\left(\gamma^{\prime}\right)_{*} M\left(1, z_{0}\right)^{-1} \\
& =\left(\rho^{-1}\right)_{*}\left(p M\left(1, z_{0}\right)^{-1}+i q M\left(1+z_{0}, 1-z_{0}\right)^{-1}\right) \\
& =p M(1, z)^{-1}+i q M(1+z, 1-z)^{-1}
\end{aligned}
$$

where $(p, q)$ is the first row of the matrix determined by $\gamma^{\prime}$ and the homomorphism (4.14).

The following lemma can be proved by a standard discussion which will be reviewed in the next section.

Lemma 4.1 The set of the first rows of matrices belonging to $\Gamma$ equals the set $\{(p, q) ; p$ and $q$ are relatively prime integers such that $p \equiv 1 \bmod$ 4 and $q \equiv 0 \bmod 4\}$.

Assuming for a moment that Lemma 4.1 is valid we have now reached the main theorem of Cox [1] with an alternative proof via Theorem 3.7 and (4.15). Our version is slightly finer than Cox's (as in Theorem 2.9) with respect to the condition of $a$ and $b$. More precisely the condition $|a| \geq|b|$ can be replaced with a weaker one:

$$
\frac{b}{a} \notin\{z \in \mathbb{R} ; z<-1\} \text {. }
$$

Theorem 4.2 Let $a$ and $b$ satisfy $a \pm b \neq 0, a b \neq 0$ and (4.16). Then $\tau$ belongs to $\mathfrak{M}(a, b)$ if and only if

$$
\frac{1}{\tau}=\frac{p}{M(a, b)}+i \frac{q}{M(a+b, a-b)}
$$

where $p$ and $q$ are arbitrary relatively prime integers satisfying

$$
p \equiv 1 \bmod 4 \quad \text { and } \quad q \equiv 0 \bmod 4 .
$$

Now we consider the case where the condition (4.16) fails. This condition is necessary there since $M(1, z)$ and $M(1+z, 1-z)$ are continuous on the real interval $(-\infty,-1)$ respectively from below and from above. This irregularity is a reflection of the inequality $\operatorname{Im}\left(b_{n} / a_{n}\right)>0$ in $(2.2)$ concerning the definition of the right choice. In fact this was put somewhat randomly, 
and instead of (2.2) the definition could have been

$$
\operatorname{Re}\left(\frac{b_{n}}{a_{n}}\right)>0, \text { or } \operatorname{Re}\left(\frac{b_{n}}{a_{n}}\right)=0, \quad \operatorname{Im}\left(\frac{b_{n}}{a_{n}}\right)<0,
$$

without any essential changes in our subsequent argument. (4.17) can lead to another arithmetic-geometric mean that differs slightly from the simplest mean.

Definition 4.3 Let $\left\{\left(a_{n}, b_{n}\right)\right\}$ be an agm-sequence for $(a, b)$. Suppose that $\left(a_{n}, b_{n}\right)$ satisfies $(4.17)$ for every $n \geq 1$. We denote by $M_{c}(a, b)$ the limit of the sequence $\left\{a_{n}\right\}$ (or $\left.\left\{b_{n}\right\}\right)$.

Obviously

$$
M(1, z)=M_{c}(1, z), \quad z \in \mathbb{C}_{0} \backslash\{t \in \mathbb{R} ; t<0\} .
$$

Furthermore, $M_{c}(1, z)$ is continuous from above on the real interval $(-\infty,-1)$ and from below on the real interval $(-1,0)$. Therefore $M_{c}(1+$ $z, 1-z)$ is continuous from below on $(-\infty,-1)$. For any $z \in \mathbb{C}_{0}$, then, there exists a continuous curve $\rho$ in $\mathbb{C}_{0}$ joining $z$ with a point in the real interval $(0,1)$ such that both $M(1, z)$ and $M_{c}(1+z, 1-z)$ are continuous on $\rho$. The same reasoning used to prove Theorem 4.2 now applies to get

Theorem 4.4 Let $a$ and $b$ satisfy $a \pm b \neq 0, a b \neq 0$. Then $\tau$ belongs to $\mathfrak{M}(a, b)$ if and only if

$$
\frac{1}{\tau}=\frac{p}{M(a, b)}+i \frac{q}{M_{c}(a+b, a-b)}
$$

where $p$ and $q$ are arbitrary relatively prime integers satisfying

$$
p \equiv 1 \bmod 4 \text { and } q \equiv 0 \bmod 4 .
$$

\section{A modular group}

This section is devoted to a proof of Lemma 4.1. We begin with the introduction of the principal congruence subgroup of level 2 :

$$
\Gamma(2):=\left\{\mu \in S L(2, \mathbb{Z}) ; \mu \equiv\left(\begin{array}{ll}
1 & 0 \\
0 & 1
\end{array}\right) \bmod 2\right\} .
$$

In the previous section we defined $\Gamma$ as the subgroup of $S L(2 . \mathbb{Z})$ generated by $V, U^{-2}$ and $U V U^{-1}$. It is clear that $\Gamma \subset \Gamma(2)$. The following lemma is 
contained in Cox [1, Lemma 2.5].

Lemma 5.1 $\Gamma(2)$ is generated by $-1, U$ and $V$.

The following subgroup of $\Gamma(2)$ will play an important role:

$$
\Gamma_{2}(4)=\left\{\left(\begin{array}{cc}
p & q \\
r & s
\end{array}\right) \in \Gamma(2) ; p \equiv s \equiv 1 \bmod 4, q \equiv 0 \bmod 4\right\} .
$$

Proposition $5.2 \quad \Gamma_{2}(4)$ is generated by $V, U^{-2}$ and $U V U^{-1}$. That is, $\Gamma=\Gamma_{2}(4)$.

Proof. The inclusion $\Gamma \subset \Gamma_{2}(4)$ is obvious. We will therefore prove the converse $\Gamma_{2}(4) \subset \Gamma$. Let $\mu \in \Gamma_{2}(4) \subset \Gamma(2)$. Lemma 5.1 allows us to write

$$
\mu= \pm U^{a_{1}} V^{b_{1}} \cdots U^{a_{m}} V^{b_{m}}
$$

with some integers $a_{j}$ and $b_{j}, 1 \leq j \leq m$. We may write $U^{a_{1}} V^{b_{1}}=$ $\left(U^{2}\right)^{a_{1} / 2} V^{b_{1}} \in \Gamma$, if $a_{1}$ is even and $U^{a_{1}} V^{b_{1}}=U^{a_{1}-1}\left(U V U^{-1}\right)^{b_{1}} U$, if $a_{1}$ is odd. In either case there exists $\gamma \in \Gamma$ such that

$$
\gamma \mu= \pm U^{a_{2}^{\prime}} V^{b_{2}} \cdots U^{a_{m}} V^{b_{m}} .
$$

Repeating the above argument we get some $\gamma^{\prime} \in \Gamma$ such that $\gamma^{\prime} \mu$ is equal to one of \pm 1 and $\pm U$. Among these, however, only 1 belongs to $\Gamma_{2}(4)$. Therefore $\gamma^{\prime} \mu=1$, which says $\mu \in \Gamma$.

Lastly, in order to prove Lemma 4.1, let $p$ and $q$ be arbitrary relatively prime integers such that $p \equiv 1 \bmod 4$ and $q \equiv 0 \bmod 4$. There exist $r, s \in \mathbb{Z}$ such that $p s-q r=1$. This equality implies $p s \equiv 1 \bmod 4$ and hence $s \equiv$ $1 \bmod 4$. If $r$ is even we have

$$
\left(\begin{array}{ll}
p & q \\
r & s
\end{array}\right) \in \Gamma_{2}(4)
$$

If $r$ is odd, then $p+r$ becomes even and

$$
\left(\begin{array}{cc}
p & q \\
p+r & q+s
\end{array}\right) \in \Gamma_{2}(4) .
$$

Since the converse part of Lemma 4.1 is clear, its proof is now complete. 


\section{Proof of Theorem 1}

If $\left\{\left(a_{n}, b_{n}\right)\right\}$ is an agm-sequence for $(a, b)$, then the same sequence $\left\{\left(a_{n}, b_{n}\right)\right\}$ except for $\left(a_{0}, b_{0}\right)=(b, a)$ is an agm-sequence for $(b, a)$. Therefore in proving Theorem 1 we may assume $|a| \geq|b|$ without loss of generality. Furthermore due to the homogeneity (3.1) we may assume that $a=1$ and $b=z_{0}$ with $\left|z_{0}\right| \leq 1$.

Let $\left\{\left(a_{n}(w), b_{n}(w)\right)\right\}$ and $\left\{\left(a_{n}^{\prime}(w), b_{n}^{\prime}(w)\right)\right\}$ be agm-sequences for $(1, w)$ with $w$ in a neighborhood of $z_{0}$ such that $a_{n}(w), b_{n}(w), a_{n}^{\prime}(w)$ and $b_{n}^{\prime}(w)$ are single-valued, holomorphic functions for every $n$, satisfying $\left\{\left(a_{n}\left(z_{0}\right), b_{n}\left(z_{0}\right)\right)\right\}=\left\{\left(a_{n}, b_{n}\right)\right\}$ and $\left\{\left(a_{n}^{\prime}\left(z_{0}\right), b_{n}^{\prime}\left(z_{0}\right)\right)\right\}=\left\{\left(a_{n}^{\prime}, b_{n}^{\prime}\right)\right\}$. Suppose that their respective limits $\tau_{1}(w)$ and $\tau_{2}(w)$, which are also holomorphic, coincide at $z_{0}: \tau_{1}\left(z_{0}\right)=\tau_{2}\left(z_{0}\right)$. We wish to prove $\left(a_{n}(w), b_{n}(w)\right)=$ $\left(a_{n}^{\prime}(w), b_{n}^{\prime}(w)\right)$ for every $n$.

In view of Theorem 4.2 (or rather its proof) one can write with some integers $p_{j}$ and $q_{j}, j=1,2$,

$$
\begin{aligned}
& \frac{1}{\tau_{1}(w)}=\frac{p_{1}}{M(1, w)}+i \frac{q_{1}}{M(1+w, 1-w)}, \\
& \frac{1}{\tau_{2}(w)}=\frac{p_{2}}{M(1, w)}+i \frac{q_{2}}{M(1+w, 1-w)}
\end{aligned}
$$

for $w$ in a neighborhood $U$ of $z_{0}$ if $z_{0} \notin(-1,0)$ or in a half neighborhood $U \cap\{z ; \operatorname{Im} z \geq 0\}$ of $z_{0}$ if $z_{0} \in(-1,0)$. In what follows $w$ stands for any point lying in this (half) neighborhood.

Now (4.6) and (4.7) show that $M(1, w)^{-1}$ and $i M(1+w, 1-w)^{-1}$ are different periods of the elliptic curve (4.8). Therefore they are linearly independent over $\mathbb{R}$ (see e.g. [6]). From the equality of $\tau_{1}$ and $\tau_{2}$ at $z_{0}$ it follows then that $\left(p_{1}, q_{1}\right)=\left(p_{2}, q_{2}\right)$. This in turn implies that $\tau_{1}(w)=\tau_{2}(w)$ as holomorphic germs.

It is not clear yet whether both agm-sequences $\left\{\left(a_{n}(w), b_{n}(w)\right)\right\}$ and $\left\{\left(a_{n}^{\prime}(w), b_{n}^{\prime}(w)\right)\right\}$ are identical or not. However, a repeated use of Proposition 3.6 shows that there exists a closed path $\rho_{1}$ through $w$ such that

$$
\begin{aligned}
\left(\rho_{1}\right)_{*}\left\{\left(a_{n}(w), b_{n}(w)\right)\right\} & =\left\{\left(c_{n}(w), d_{n}(w)\right)\right\} \\
\left(\rho_{1}\right)_{*} \tau_{1}(w) & =M(1, w) .
\end{aligned}
$$

Here $\left\{\left(c_{n}(w), d_{n}(w)\right)\right\}$ is the agm-sequence for $(1, w)$ consisting only of right choices with its limit $M(1, w)$. Similarly we have another closed path $\rho_{2}$ 
through $w$ such that

$$
\begin{aligned}
\left(\rho_{2}\right)_{*}\left\{\left(a_{n}^{\prime}(w), b_{n}^{\prime}(w)\right)\right\} & =\left\{\left(c_{n}(w), d_{n}(w)\right)\right\} \\
\left(\rho_{2}\right)_{*} \tau_{2}(w) & =M(1, w) .
\end{aligned}
$$

Since $\tau_{1}=\tau_{2}$, we have

$$
\left(\rho_{2} \rho_{1}^{-1}\right)_{*} M(1, w)=M(1, w),
$$

although the same analytic continuation may change $\left\{\left(c_{n}(w), d_{n}(w)\right)\right\}$ to a different agm-sequence.

In order to prove that this is not the case, we consider a homomorphism induced from (4.14):

$$
\mu: \pi_{1}\left(\mathbb{C}_{0}, w\right) \longrightarrow S L(2, \mathbb{Z}) .
$$

This can be decomposed into the following maps:

$$
\pi_{1}\left(\mathbb{C}_{0}, w\right) \stackrel{\mu_{1}}{\longrightarrow} \pi_{1}\left(\mathbb{C}_{0}, z_{1}\right) \stackrel{\mu_{2}}{\longrightarrow} \pi_{1}\left(\mathbb{C}_{1}, \lambda_{1}\right) \stackrel{\mu_{3}}{\longrightarrow} S L(2, \mathbb{Z}) .
$$

Here $z_{1}$ is a point $\in(0,1)$ in the real axis, $\lambda_{1}=1 /\left(1-z_{1}^{2}\right), \mu_{2}=\varphi_{*}$ in $(4.3)$ and $\mu_{3}$ is defined by the relations (4.10), namely

$$
\mu_{3}\left(\delta_{1}\right)=U^{-1}, \quad \mu_{3}\left(\delta_{\infty}\right)=V^{-1}
$$

with the generators $\delta_{1}$ and $\delta_{\infty}$ of $\pi_{1}\left(\mathbb{C}_{1}, \lambda_{1}\right) . \mu_{1}$ is derived from the correspondence: $\gamma \mapsto \sigma \gamma \sigma^{-1}$ for any cycle $\gamma$ through $w$, where $\sigma$ is a path in $\mathbb{C}_{0}$ connecting $w$ with $z_{1}$ such that both $M(1, z)$ and $M(1+z, 1-z)$ are continuous on $\sigma$.

The relations (4.15) and (6.1) imply that $\mu\left(\rho_{2} \rho_{1}^{-1}\right)$ is a matrix of the form

$$
\mu\left(\rho_{2} \rho_{1}^{-1}\right)=\left(\begin{array}{ll}
1 & 0 \\
* & *
\end{array}\right) \in \Gamma_{2}(4),
$$

which must be $V^{m}$ for some $m \in \mathbb{Z}$.

As in Section 4 let $\gamma_{1}$ be a cycle through $z_{1}$ encircling the point 1 once counterclockwise but leaving the points 0 and -1 outside. We put $\sigma_{1}=\sigma^{-1} \gamma_{1} \sigma$. From $(4.11) \sim(4.13)$ we know $\mu\left(\sigma_{1}\right)=V$.

On the other hand it is clear that the maps $\mu_{1}$ and $\mu_{2}$ are injective . It is also known that $\mu_{3}$ is injective (see the remark below). Hence $\mu$ is 
injective. This implies that for some $m \in \mathbb{Z}$

$$
\rho_{2} \rho_{1}^{-1}=\sigma_{1}^{m} .
$$

Proposition 3.7 asserts that $\left(\sigma_{1}\right)_{*}\left\{\left(c_{n}(w), d_{n}(w)\right)\right\}=\left\{\left(c_{n}(w), d_{n}(w)\right)\right\}$. Therefore we have

$$
\begin{aligned}
\left\{\left(a_{n}(w), b_{n}(w)\right)\right\} & =\left(\rho_{1}^{-1}\right)_{*}\left\{\left(c_{n}(w), d_{n}(w)\right)\right\} \\
& =\left(\rho_{2}^{-1} \sigma_{1}^{m}\right)_{*}\left\{\left(c_{n}(w), d_{n}(w)\right)\right\} \\
& =\left\{\left(a_{n}^{\prime}(w), b_{n}^{\prime}(w)\right)\right\} .
\end{aligned}
$$

Putting in particular $w=z_{0}$, we have completed the proof of Theorem 1 .

Remark 6.1. The fact that the homomorphism $\mu_{3}$ is injective can be proved in the framework of the Gauss-Schwarz theory of monodromy representations (see e.g. Yoshida [8, p54]). Or we may refer to Lehner [3, Chap. XI, $3 \mathrm{D}$ Theorem], in which it is proved that $\Gamma(2) / \pm 1$ is a free group generated by $U$ and $V$. It is then obvious that $\mu_{3}$ with its assignment (6.3) is injective.

\section{References}

[1] Cox D.A., The arithmetic-geometric mean of Gauss. Enseign. Math., 30 (1984), 275-330.

[2] Geppert H., Zur Theorie des arithmetisch-geometrischen Mittels. Math. Annalen 99 (1928), 162-180.

[3] Lehner J., Discontinuous groups and automorphic functions. American Mathematical Society, Providence, Rhode Island (1966).

[4] Nishiwada K., A holomorphic structure of the arithmetic-geometric mean of Gauss. Proc. Japan Acad. 64 (1988), 322-324.

[5] Nishiwada K., Holomorphic structure of the arithmetic-geometric mean of Gauss, II. Proc. Japan Acad. 70 (1994), 119-122.

[6] Rauch H.E. and A. Lebowitz, Elliptic functions, theta functions and Riemann surfaces. Williams \& Wilkins, Baltimore (1973).

[7] von David L., Arithmetisch-geometrisches Mittel und Mudulfunktion. J. für die Reine u. Ang. Math. 159 (1928), 154-170.

[8] Yoshida, M., Fuchsian differential equations. Vieweg \& Sohn, Braunschweig/Wiesbaden (1987). 
Division of Mathematics

Department of Fundamental Sciences

Faculty of Integrated Human Studies

Kyoto University

Kyoto 606-01, Japan

E-mail: kimi@math.h.kyoto-u.ac.jp 